Pacific

Journal of

Mathematics

DIAGONALIZABLE DERIVATIONS OF FINITE-DIMENSIONAL ALGEBRAS II

Daniel R. Farkas, Edward L. Green, and Eduardo N. Marcos 


\title{
DIAGONALIZABLE DERIVATIONS OF FINITE-DIMENSIONAL ALGEBRAS II
}

\author{
Daniel R. Farkas, Edward L. Green, and Eduardo N. Marcos
}

\begin{abstract}
When an algebra is graded by a group, any additive character of the group induces a diagonalizable derivation of the ring. This construction is studied in detail for the case of a path algebra modulo relations and its fundamental group. We describe an injection of the character group into the first cohomology group following Assem-de la Peña. Rather general conditions are determined, in this context, which guarantee that a diagonalizable derivation is induced from the fundamental group.
\end{abstract}

This paper is the second installment in a series devoted to diagonalizable derivations. Suppose that $R$ is a finite-dimensional algebra over the field $k$. We will denote by $\operatorname{Der}(R)$ the space of $k$-algebra derivations from $R$ to itself. Diagonalizable derivations arise naturally whenever $R$ is graded by a group $H$. Indeed, every additive character $\chi \in \operatorname{Hom}\left(H, k^{+}\right)$can be assigned a derivation $D_{\chi} \in \operatorname{Der}(R)$ according to the rule

$$
D_{\chi}(r)=\chi(g) r
$$

for every $r \in R$ in the homogeneous component of "degree" $g \in H$. Obviously, $D_{\chi}$ is diagonalizable. Conversely, if $D$ is a diagonalizable derivation of the $k$-algebra $S$ and $H$ is the additive subgroup of $k$ generated by the eigenvalues of $D$ then, for the inclusion map $\iota: H \rightarrow k^{+}$, we have $D=D_{\iota}$.

In our first paper $[$ FGGM], we proved that the span of all diagonalizable derivations of $R$ comprise a Lie ideal of $\operatorname{Der}(R)$ whenever $k$ has characteristic zero or is algebraically closed of positive characteristic. This result turned out to be a powerful tool in describing what we called spanned-bysplit derivations (i.e., those which are sums of diagonalizable derivations) in several classes of algebras. In what follows, we shall use the notation

$\operatorname{SPDer}(R)$

for the subspace of $\operatorname{Der}(R)$ consisting of spanned-by-split derivations.

This paper describes some of the examples which motivated our original paper. Consider $R$ presented as $k \Gamma / I$, a path algebra modulo relations, graded by the fundamental group $\pi_{1}(\Gamma, I)$. In section one, we review and 
clarify notions of fundamental group. The second section is devoted to tightening two results of Assem and de la Peña:

- The map which assigns to each character in $\operatorname{Hom}\left(\pi_{1}(\Gamma, I), k^{+}\right)$a derivation in $\operatorname{SPDer}(k \Gamma / I)$ is injective.

- The induced map of $\operatorname{Hom}\left(\pi_{1}(\Gamma, I), k^{+}\right)$to $H^{1}(k \Gamma / I)$ is injective.

Next we give a partial characterization of those diagonalizable derivations which arise in the form $D_{\chi}$. Essentially, we require that the underlying algebra have its radical stabilized by the derivation and that the algebra of constants for the derivation be indecomposable.

We close with a short section placing our constructions in the context of Hopf algebras.

\section{Fundamental Groups.}

Let $\Gamma$ be a finite connected directed graph. Temporarily forget about the orientation of arrows, obtaining the undirected graph $\Gamma^{\mathrm{un}}$. If $\alpha$ is a walk from vertex $x$ to vertex $y$ and $\beta$ is a walk from vertex $y$ to vertes $z$ then the concatenation $\alpha \beta$ is a walk from $x$ to $z$ and the reverse $\alpha^{-1}$ is a walk from $y$ to $x$. Consider the "homotopy relation", the smallest equivalence relation on walks which is compatible with right and left concatenation (whenever they make sense) and for which $\sigma \sigma^{-1}$ is equivalent to the trivial walk at $w$ for any walk $\sigma$ beginning at $w$. For a fixed vertex $x$, the classes of closed walks from $x$ to itself comprise the fundamental group, $\pi_{1}(\Gamma)$. (Different choices of $x$ yield isomorphic groups.)

We will need a more traditional description of the fundamental group. Fix a vertex $v$, the "base point". For each vertex $w \in \Gamma$, choose a walk $\gamma_{v, w}$ from $v$ to $w$ in the underlying undirected graph $\Gamma^{\mathrm{un}}$; we require that $\gamma_{v, v}$ be the empty walk from $v$ to itself. The set

$$
\gamma=\left\{\gamma_{v, w} \mid w \text { is a vertex }\right\}
$$

will be referred to as a choice of parade data. If $f$ is any walk in $\Gamma^{u n}$ from $x$ to $y$ then we define

$$
c_{\gamma}(f)=\gamma_{v, x} f \gamma_{v, y}^{-1}
$$

the walk which begins at $v$, takes the parade route to $x$, follows $f$ from $x$ to $y$, and then reverses the parade route to return to $v$ from $y$. Observe that if $g$ is already a closed walk from $v$ to $v$ then $c_{\gamma}(g)=g$. Also, every $c_{\gamma}(f)$ lies in the subgroup generated by

$$
\left\{c_{\gamma}(a) \mid a \text { is an arrow in } \Gamma\right\} .
$$

Suppose the parade walk $\gamma_{v, w}$ is a sequence

$$
a_{1}^{\varepsilon(1)}, a_{2}^{\varepsilon(2)}, \ldots, a_{t}^{\varepsilon(t)}
$$


of edges where each $a_{j}$ is an arrow in $\Gamma$ and $\varepsilon(j)= \pm 1$ according to whether the original orientation is preserved or reversed in the walk. Then

$$
c_{\gamma}\left(a_{1}\right)^{\varepsilon(1)} c_{\gamma}\left(a_{2}\right)^{\varepsilon(2)} \cdots c_{\gamma}\left(a_{t}\right)^{\varepsilon(t)}=1 .
$$

We call the word on the left-hand side of the last equation a parade walk relator.

Given fixed parade data $\gamma$, the earlier remark about closed walks through $v$ implies that $\pi_{1}(\Gamma)$ is generated by $\left\{c_{\gamma}(a) \mid a\right.$ is an arrow $\}$. It is less obvious that $\pi_{1}(\Gamma)$ is the free group on the formal symbols $c_{\gamma}(a)$ modulo the parade walk relators. (Let $F_{\gamma}$ be the free group on arrows modulo the parade walk relations for $\gamma$. The obvious map from walks in $\Gamma^{\text {un }}$ to $F_{\gamma}$ respects the homotopy equivalence relation. If we regard $\pi_{1}(\Gamma)$ as the group of equivalence classes of closed walks through $v$ then the restriction of the factored map is a group homomorphism $\phi: \pi_{1}(\Gamma) \rightarrow F_{\gamma}$. For any arrow $a$ from $x$ to $y$,

$$
\begin{aligned}
\phi\left(c_{\gamma}(a)\right) & =\phi\left(\gamma_{v, x} a \gamma_{v, y}^{-1}\right) \\
& =\phi\left(\gamma_{v, x}\right) \phi(a) \phi\left(\gamma_{v, y}\right)^{-1} \\
& =\phi(a) \\
& =\bar{a}
\end{aligned}
$$

where $\bar{a}$ is the image of the symbol $a$ in $F_{\gamma}$. Thus $\phi$ is surjective. But $\pi_{1}(\Gamma)$ is generated by the collection of all such $c_{\gamma}(a)$ and they are subject to the parade walk relations. It follows that $\phi$ is an isomorphism.) When the context is clear, we drop the subscript $\gamma$.

Suppose that $I$ is an ideal of the path algebra $k \Gamma$ and that $I$ is generated as an ideal by a set of relations $\rho$. The fundamental group $\pi_{1}(\Gamma, \rho)$ will turn out to be a certain image of $\pi_{1}(\Gamma)$. While it is possible to describe $\pi_{1}(\Gamma, \rho)$ abstractly $([\mathbf{S}])$, we will assume that $\pi_{1}(\Gamma)$ is already described using parade data $\gamma$. Let $N(\rho)$ be the normal subgroup of $\pi_{1}(\Gamma)$ generated by $c_{\gamma}(p) c_{\gamma}\left(q^{-1}\right)$ as $p$ and $q$ range over all paths in the support of the same member of $\rho$. Then

$$
\pi_{1}(\Gamma, \rho)=\pi_{1}(\Gamma) / N(\rho) .
$$

We denote the canonical homomorphism from $\pi_{1}(\Gamma)$ to $\pi_{1}(\Gamma, \rho)$ (which depends on $\gamma$ ) by $\xi$.

A choice of parade data $\gamma$ induces a $\pi_{1}(\Gamma, \rho)$-grading on $k \Gamma / I$. Explaining this gives us the opportunity to introduce the useful notion of weight $([\mathbf{G}])$. Suppose that $\Gamma$ is a finite directed graph and $H$ is a group. A weight function for $\Gamma$ with values in $H$ is an assignment $W$ from the arrows of $\Gamma$ to $H$. If we extend $W$ multiplicatively so that vertices have weight $1 \in H$ then the domain of the extension (also called $W$ ) consists of all directed paths in $\Gamma$. The weight function now induces an $H$-grading on $k \Gamma$. We say that an ideal $I$ of $k \Gamma$ is homogeneous for $W$ provided it is homogeneous with respect to this grading. For such an ideal, the weight induces a grading on $k \Gamma / I$. In 
the case of fundamental groups, we can consider the weight with values in $\pi_{1}(\Gamma, \rho)$ which sends an arrow $a$ to $\xi\left(c_{\gamma}(a)\right)$. The ideal $I$ is homogeneous by the construction of $N(\rho)$.

Unfortunately, $\pi_{1}(\Gamma, \rho)$ is dependent on the choice of relations for $I$. This can be remedied as follows. We say that a nonzero element $r \in I$ is support minimal if it cannot be written as a sum of two elements of $I$, each of whose supports are proper subsets of the support of $r$. (If $\sum \alpha_{p} p \in I$ is support minimal where the sum runs over paths $p$ with scalars $\alpha_{p} \neq 0$ then we cannot erase any summands and maintain the subsum in $I$.) It is an immediate consequence of the next proposition that the fundamental group is the same for any two choices of $\rho$ which consist of support minimal relations; this common group is denoted $\pi_{1}(\Gamma, I)$.

Proposition 1.1. Let $\rho$ be any generating set for $I$ and suppose that $s \in I$ is support-minimal. Then $c(p) c(q)^{-1} \in N(\rho)$ for all $p$ and $q$ in the support of $s$.

Proof. By definition, if $r \in \rho$ and $\sigma$ is a path in the support of $r$ then

$$
c(\tau) \equiv c(\sigma) \quad(\bmod N(\rho))
$$

for all $\tau$ in the support of $r$. We will abuse notation and write

$$
\operatorname{supp}(r) \equiv c(\sigma) \quad(\bmod N(\rho)) .
$$

For any two paths $\alpha$ and $\beta$, we then have

$$
\operatorname{supp}(\alpha r \beta) \equiv c(\alpha \sigma \beta) \quad(\bmod N(\rho)) .
$$

An arbitrary $s \in I$ is a linear combination of expressions $\alpha r \beta$ for paths $\alpha, \beta$ and for $r \in \rho$. Given $d \in \pi_{1}(\Gamma, \rho)$, set $s(d)$ to be the subcombination of all those $\alpha r \beta$ whose support lies in $d$, regarded as a coset. Then

$$
s=\sum_{d} s(d)
$$

with $s(d) \in I$ and the supports of the $s(d)$ pair-wise disjoint. Hence if $s$ is support-minimal it must be equal to a single $s(d)$.

We mention one last time that we will only be able to speak about a $\pi_{1}(\Gamma, I)$-grading of $k \Gamma / I$ in the presence of parade data $\gamma$. Thus if $\Psi \in$ $\operatorname{Hom}\left(\pi_{1}(\Gamma, I), k^{+}\right)$, then the induced derivation defined in the introduction depends on some choice of $\gamma$ and, so, will frequently be written $D_{\Psi, \gamma}$.

\section{Injectivity Theorems.}

In this section, make the standing assumption that $I$ is an admissible ideal of $k \Gamma$. (That is, we assume that $k \Gamma / I$ is finite-dimensional and $I$ lies inside the square of the ideal generated by all arrows.) 
Proposition 2.1. Choose parade data for the connected directed graph $\Gamma$. Then $D_{\Psi}$ is diagonalizable for every $\Psi \in \operatorname{Hom}\left(\pi_{1}(\Gamma, I), k^{+}\right)$. Moreover the map

$$
\operatorname{Hom}\left(\pi_{1}(\Gamma, I), k^{+}\right) \rightarrow \operatorname{SPDer}(k \Gamma / I)
$$

is injective.

Proof. It is obvious that $D_{\Psi}$ is diagonalizable, so $\operatorname{Hom}\left(\pi_{1}(\Gamma, I), k^{+}\right)$maps into $\operatorname{SPDer}(k \Gamma)$. Thus the issue is injectivity. By the standing assumption, every arrow in $\Gamma$ survives modulo $I$. Since $\pi_{1}(\Gamma, I)$ is generated by

$$
\{\xi(c(a)) \mid a \text { is an arrow }\},
$$

we see that $\pi_{1}(\Gamma, I)$ is generated by degrees which genuinely occur. (In the literature, the grading is sometimes referred to as full.)

However if $R$ is any $H$-graded algebra and

$$
\{h \in H \mid \text { the } h \text {-component of } R \text { is not } 0\}
$$

generates $H$ then the map $\operatorname{Hom}\left(H, k^{+}\right) \rightarrow \operatorname{Der}(R)$ is always injective.

The next theorem provides a more significant injectivity result, which is based on a similar statement of Assem-de la Peña ([AP]).

Theorem 2.1. Suppose $\chi \in \operatorname{Hom}\left(\pi_{1}(\Gamma, I), k^{+}\right)$and fix parade data for $\pi_{1}(\Gamma)$. If the associated derivation $D_{\chi}$ on $k \Gamma / I$ is inner then $\chi=0$. Hence the induced map

$$
\operatorname{Hom}\left(\pi_{1}(\Gamma, I), k^{+}\right) \rightarrow H^{1}(k \Gamma / I)
$$

is injective.

Proof. We shall write $\Lambda=k \Gamma / I$. Then $\Lambda=\Lambda_{0} \oplus \operatorname{rad} \Lambda$ where we identify $\Lambda_{0}$ with $(k \Gamma)_{0}$ : A commutative subalgebra with basis consisting of orthogonal idempotents $e(w)$, one for each vertex $w$ of $\Gamma$.

For $s=\sum_{w} \lambda_{w} e(w) \in \Lambda_{0}$ we compute ad $s$. If $\bar{m} \in \Lambda$ is the image of a path $m$ in $\Gamma$ from vertex $x$ to vertex $y$ then

$$
(\operatorname{ad} s)(\bar{m})=\left(\lambda_{x}-\lambda_{y}\right) \bar{m} \text {. }
$$

Thus ad $s$ is always diagonalizable and all images of paths are among its eigenvectors.

Now suppose that $\chi \in \operatorname{Hom}\left(\pi_{1}(\Gamma, I), k^{+}\right)$and $D_{\chi}=\operatorname{ad} b$ for some $b \in \Lambda$. Set $b=s+n$ for $s \in \Lambda_{0}$ and $n \in \operatorname{rad} \Lambda$. The image $\bar{m}$ of every path is an eigenvector for $D_{\chi}$ corresponding to eigenvalue $(\chi \circ \xi)(c(m))$. Thus ad $b$ is diagonalizable with a basis of eigenvectors which are images of paths. It follows that $\operatorname{ad} b$ and ad $s$ must commute. But then $(\operatorname{ad} b)-(\operatorname{ad} s)$ is diagonalizable at the same time that it is equal to ad $n$, which is nilpotent. We conclude that $D_{\chi}=$ ad $s$ for $s \in \Lambda_{0}$.

If $s=\sum_{w} \lambda_{w} e(w)$ then $(\chi \circ \xi)(c(m))=\lambda_{x}-\lambda_{y}$ for every path $m$ beginning at $x$ and ending at $y$, whose image $\bar{m}$ is nonzero. In particular, if $a$ is an 
arrow in $\Gamma$ from $x$ to $y$ and $\varepsilon= \pm 1$ the $(\chi \circ \xi)(c(\bar{a}))=\varepsilon \cdot\left(\lambda_{x}-\lambda_{y}\right)$. It follows that if $w$ is an arbitrary vertex in $\Gamma$ and

$$
a_{1}^{\varepsilon(1)}, \ldots, a_{t}^{\varepsilon(t)}
$$

is the parade walk from the base point $v$ to $w$ then

$$
(\chi \circ \xi)\left(c\left(a_{1}^{\varepsilon(1)} \cdots c\left(a_{t}\right)^{\varepsilon(t)}\right)\right)=\lambda_{v}-\lambda_{w} .
$$

On the other hand, $(\chi \circ \xi)(1)=0$. We conclude that

$$
s=\lambda_{v}\left(\sum_{w} e(w)\right)=\lambda_{v} \cdot 1 .
$$

Therefore $D_{\chi}=$ ad $s=0$.

Corollary 2.1 ([BM]). Let $\Lambda=k \Gamma / I$ be a path-monomial algebra which is finite dimensional. If $H^{1}(\Lambda, \Lambda)=0$ then $\Gamma$ is a tree.

Proof. We are assuming that the generating set $\rho$ for $I$ consists of monomials. As a consequence, $\pi_{1}(\Gamma, I)$ is a free group; it is trivial if and only if $\Gamma$ is a tree. Thus if $\Gamma$ is not a tree then $\operatorname{Hom}\left(\pi_{1}(\Gamma, I), k^{+}\right)$is nonzero. By the theorem, $H^{1}$ is nonzero.

\section{Fundamental Derivations.}

The argument presented in the previous theorem rests on the following property of the diagonalizable derivation $D_{\chi}$. For any pair of vertices $x$ and $y$ there exists an undirected walk $a_{1}^{\varepsilon(1)}, \ldots, a_{t}^{\varepsilon(t)}$ such that $\sum_{j} \varepsilon(j) D_{\chi}\left(\overline{a_{j}}\right)=0$. This property turns out to be crucial in trying to characterize those diagonalizable derivations which arise from a fundamental group.

Definition 3.1. Let $R$ be a finite-dimensional $k$-algebra. We say that a derivation $D \in \operatorname{Der}(R)$ is fundamental provided that there exists a finite directed graph $\Gamma$ and an admissible ideal $I$ of $k \Gamma$ such that $R \simeq k \Gamma / I$ and there is parade data $\gamma$ together with some $\Psi \in \operatorname{Hom}\left(\pi_{1}(\Gamma, I), k^{+}\right)$so that

$$
D=D_{\Psi, \gamma}
$$

under the identification given by the isomorphism.

There are some obvious things we can say about a fundamental derivation $D$ of $R$. First, $D$ must be diagonalizable. Indeed, the images of paths in $R$ are all eigenvectors. As another consequence, $D(\operatorname{rad} R) \subseteq \operatorname{rad} R$. Notice that the algebra $R$ is $k$-elementary, which means that $R / \operatorname{rad} R$ is a finite product of copies of $k$. More is true: The algebra complement in $k \Gamma$ to the ideal generated by all arrows, which coincides with the span of the vertex 
idempotents, survives as an algebra complement to $\operatorname{rad} R$ in $R$. Thus $\operatorname{rad} R$ has an algebra complement which lies inside the "subalgebra of constants"

$$
R^{D}=\{r \in R \mid D(r)=0\} .
$$

We shall see that these properties come close to characterizing fundamental derivations.

Lemma 3.1. Let $W$ be an $H$-valued weight on the arrows of $\Gamma$ and let $I$ be $a W$-homogeneous ideal of $k \Gamma$. Suppose that for a fixed vertex $x$ and every other vertex $y$ there exists a walk in $\Gamma^{\mathrm{un}}$,

$$
\gamma_{x, y}: b_{1}^{\varepsilon(1)}, \ldots, b_{t}^{\varepsilon(t)}
$$

from $x$ to $y$ such that

$$
W\left(b_{1}\right)^{\varepsilon(1)} \cdots W\left(b_{t}\right)^{\varepsilon(t)}=1
$$

in $H$. Then there is a homomorphism $\theta: \pi_{1}(\Gamma, I) \rightarrow H$ such that

$$
(\theta \circ \xi)\left(c_{\gamma}(a)\right)=W(a)
$$

for all arrows a.

Proof. As we remarked earlier, $\pi_{1}(\Gamma)$ is isomorphic to the free group on $\left\{c_{\gamma}(a) \mid a\right.$ is an arrow of $\left.\Gamma\right\}$ modulo the parade walk relators

$$
\left\{c_{\gamma}\left(\gamma_{x, y}\right) \mid x \neq y\right\} \text {. }
$$

Hence $W$ induces a group homomorphism $\theta: \pi_{1}(\Gamma) \rightarrow H$ such that

$$
\theta\left(c_{\gamma}(a)\right)=W(a)
$$

for all arrows $a$.

Suppose that $\rho$ is a support-minimal set of relations for the homogeneous ideal $I$. We claim that the elements of $\rho$ are homogeneous. If $r \in \rho$ write $r=\sum_{h} r_{h}$ where each $r_{h}$ is a nontrivial linear combination of paths with weight $h$. By homogeneity, each $r_{h}$ lies in $I$. But the support of $r_{h}$ is clearly a subset of the support of $r$. Hence $r=r_{h}$ for some choice of $h$.

It follows that if $p$ and $q$ are paths in the support of some $r$ in $\rho$ then $W(p)=W(q)$. Therefore $\theta$ is the identity on $N(\rho)$, the normal subgroup generated by all possible $c_{\gamma}(p) c_{\gamma}(q)^{-1}$ of this sort. We conclude that $\theta$ factors though $\pi_{1}(\Gamma, I)$.

Theorem 3.1. Assume that $I$ is an admissible ideal of $k \Gamma$. Suppose that

(a) $E$ is a diagonalizable derivation of $k \Gamma / I$ which vanishes on images of vertices and for which the images of arrows are eigenvectors, i.e., for each arrow a in $\Gamma$ there is a scalar $\omega(\bar{a})$ such that $E(\bar{a})=\omega(\bar{a}) \bar{a}$;

(b) there is a vertex $x$ such that for every other vertex $y$ there exists a walk

$$
\gamma_{x, y}: b_{1}^{\varepsilon(1)}, \ldots, b_{t}^{\varepsilon(t)}
$$


from $x$ to $y$ such that

$$
\sum_{j} \varepsilon(j) \omega\left(\overline{b_{j}}\right)=0 .
$$

Then there is some $\Psi \in \operatorname{Hom}\left(\pi_{1}(\Gamma, I), k^{+}\right)$with $E=D_{\Psi, \gamma}$ for $\gamma=\left\{\gamma_{x, y}\right\}$.

Proof. We apply the lemma with $H=k^{+}$. Since $I$ is admissible, we see that different arrows cannot have the same images in $k \Gamma / I$. Thus it makes sense to define a function $W$ on arrows via $W(a)=\omega(\bar{a})$, thereby lifting the $k^{+}$-grading to $k \Gamma$. We conclude from the lemma that there is an additive character $\Psi$ on $\pi_{1}(\Gamma, I)$ such that

$$
(\Psi \circ \xi)\left(c_{\gamma}(a)\right)=\omega(\bar{a}) .
$$

Thus the two derivations $D_{\Psi}$ and $E$ agree on the images of arrows and vertices. But these elements generate $k \Gamma / I$ as an algebra.

The next result is a close relative to Theorem 3.4 in $[\mathbf{G}]$.

Lemma 3.2. Let $R$ be a finite-dimensional $k$-elementary algebra. Assume that $D$ is a diagonalizable derivation of $R$ such that $D(\operatorname{rad} R) \subseteq \operatorname{rad} R$ and $R^{D}$ contains an algebra complement to $\operatorname{rad} R$. Then there exists a finite directed graph $\Gamma$, an admissible ideal $I$ of $k \Gamma$, and a derivation $\widetilde{D}$ of $k \Gamma$ such that

(a) $\widetilde{D}(I) \subseteq I$;

(b) $\widetilde{D}$ vanishes on the vertex idempotents of $k \Gamma$;

(c) each arrow is an eigenvector for $\widetilde{D}$;

(d) $R \simeq k \Gamma / I$ with $\widetilde{D}$ inducing $D$.

Proof. Let $e(1), \ldots, e(n)$ be orthogonal idempotents whose sum is 1 , which span a complement to $\operatorname{rad} R$, and which satisfy $D(e(j))=0$ for $j=1, \ldots, n$. Then

$$
D(e(i) \operatorname{rad} R e(j)) \subseteq e(i) \operatorname{rad} R e(j)
$$

for all $i$ and $j$. An elementary eigenspace argument using the diagonalizability of $D$ implies that the pair of $D$-stable spaces

$$
e(i) \operatorname{rad} R e(j) \cap(\operatorname{rad} R)^{2} \subseteq e(i) \operatorname{rad} R e(j)
$$

splits with a $D$-stable vector space complement $A(i, j)$.

We argue that $\{A(i, j) \mid 1 \leq i, j \leq n\}$ generates $\operatorname{rad} R$ as an algebra. Denote by $A$ the algebra generated by these subspaces. Certainly $A \subseteq \operatorname{rad} R$. If the algebras do not coincide, the nilpotence of $\operatorname{rad} R$ implies that there must be a largest $m$ such that $(\operatorname{rad} R)^{m}$ does not lie in $A$. That is, there exist $r_{1}, \ldots, r_{m} \in \operatorname{rad} R$ such that

$$
r_{1} r_{2} \cdots r_{m} \notin A \text {. }
$$


We may assume that $r_{j} \in e_{f(j)} \operatorname{rad} R e_{g(j)}$ for some choice of indices $f(j)$ and $g(j)$. Write $r_{j}=a_{j}+s_{j}$ with $a_{j} \in A(f(j), g(j))$ and $s_{j} \in(\operatorname{rad} R)^{2}$. Then

$$
r_{1} r_{2} \cdots r_{m}=a_{1} a_{2} \cdots a_{m}+z
$$

where $z \in(\operatorname{rad} R)^{m+1}$. In other words, $a_{1} \cdots a_{m}+z \in A$. We have reached the contradiction that $r_{1} \cdots r_{m} \in A$.

We can now describe $\Gamma$. Its vertices are the idempotents $e(1), \ldots, e(n)$. Choose a basis for each $A(i, j)$ which consists of eigenvectors for $D$. These basis vectors comprise the set of arrows which begin at $e(i)$ and end at $e(j)$. There is an obvious algebra map from $k \Gamma$ onto $R$. If $a$ is one of the designated eigenvectors for $D$ in $A(i, j)$ and $D(a)=\lambda a$ then we define the function $\widetilde{D}$ on the arrow $a$ by $\widetilde{D}(a)=\lambda a$. It is easy to see that $\widetilde{D}$ extends uniquely to a derivation of $k \Gamma$ which vanishes on vertex idempotents.

The lemma follows with $I$ the kernel of the map $k \Gamma \rightarrow R$.

Theorem 3.2. Let $R$ be a finite-dimensional k-elementary algebra. Suppose that $D$ is a diagonalizable derivation of $R$ with $D(\operatorname{rad} R) \subseteq \operatorname{rad} R$. Suppose, further, that $R^{D}$ contains an algebra complement to $\operatorname{rad} R$ and that $R^{D}$ is indecomposable as an algebra. Then $D$ is fundamental.

Proof. We carry over all of the notation in the previous lemma. Define a new graph $G$ whose vertices are $e(1), \ldots e(n)$ (the vertices of $\Gamma$ ) and construct an arrow from $e(i)$ to $e(j)$ provided $e(i) R^{D} e(j) \neq 0$. The sum of the $e(h)$ over all those vertex idempotents in a connected component of $G$ is a central idempotent of $R^{D}$. Since $R^{D}$ is indecomposable, $G$ is connected.

We claim that if $e(i) R^{D} e(j) \neq 0$ then there is a path $m$ in $\Gamma$ such that $m$ begins at $e(i)$, ends at $e(j)$, and $D(\bar{m})=0$. To see this, suppose that $e(i) x e(j) \neq 0$ for $x \in R^{D}$. Write $x=\sum \alpha_{p} \bar{p}$ with $\alpha_{p}$ a nonzero scalar and $\bar{p}$ the image in $R$ of a path $p$ in $k \Gamma$ which begins at $e(i)$ and ends at $e(j)$. We may assume that the $\bar{p}$ which appear in the sum are linearly independent in $R$. Each such $\bar{p}$ is an eigenvector for $D$. Since eigenvectors for distinct eigenvalues are linearly independent, we conclude that $D(\bar{p})=0$ for every $\bar{p}$ which appears.

We put the previous two paragraphs together. For each $i \neq j$ there is a walk from $e(i)$ to $e(j)$ in $G$ with edge sequence

$$
g_{1}^{\varepsilon(1)}, g_{2}^{\varepsilon(2)}, \ldots, g_{v}^{\varepsilon(v)} .
$$

This walk gives rise to an "expanded" walk

$$
m_{1}^{\varepsilon(1)}, m_{2}^{\varepsilon(2)}, \ldots, m_{v}^{\varepsilon(v)}
$$

from $e(i)$ to $e(j)$ in $\Gamma$, where each $m_{d}$ is a path with the same endpoints as $g_{d}$ and $D\left(\overline{m_{d}}\right)=0$. If we rewrite the second walk as

$$
a_{1}^{\eta(1)}, a_{2}^{\eta(2)}, \ldots, a_{t}^{\eta(t)}
$$


for arrows $a_{i}$ in $\Gamma$ then

$$
\sum \eta(i) D\left(\overline{a_{i}}\right)=0
$$

(The point is that if the sum of the eigenvalues along a path $m_{d}$ is zero then the same is true for the sum of the negatives of those eigenvalues in reverse order along the path.) For each $2 \leq j \leq n$ pick such a walk $\gamma_{1, j}$ from the base point $e(1)$ to $e(j)$.

According to Theorem 3.1, there exists some $\Psi \in \operatorname{Hom}\left(\pi_{1}(\Gamma, I), k^{+}\right)$such that $D=D_{\Psi, \gamma}$.

Corollary 3.1. Assume that $k$ is an algebraically closed field of characteristic zero and $R$ is a finite-dimensional local $k$-algebra. Then every diagonalizable derivation of $R$ is fundamental.

Proof. Since $k$ is algebraically closed and $R$ is local, we have

$$
R / \operatorname{rad} R \simeq k .
$$

It follows that $R^{D}$ is local, and so, indecomposable. Finally, it is well known that $D(\operatorname{rad} R) \subseteq \operatorname{rad} R$ for any $D \in \operatorname{Der}(R)$, by virtue of chark $=0$.

\section{Hopf Algebras.}

We end with a hint that there may be other classes of derivations beside diagonalizable ones for which an interesting theory exists. Every diagonalizable derivation of a $k$-algebra corresponds to a group grading by a subgroup of the additive group $k^{+}$. Equivalently, every diagonalizable derivation has the form $D_{\chi}$ where $\chi \in \operatorname{Hom}\left(G, k^{+}\right)$for some group $G$ which grades the algebra. A group grading for an algebra $R$ is an example of an $H$-comodule algebra action on $R$, where $H$ is a Hopf algebra. (In the special case, $H=k G$ with the standard Hopf structure.) In general, if $H$ is any Hopf algebra then $R$ is an $H$-comodule algebra provided that $R$ is a left $H$-comodule, via

$$
\lambda: R \rightarrow H \otimes R
$$

(so $\lambda(a)=\sum a_{0} \otimes a_{1}$ for $a \in R$ ) and

$$
\begin{aligned}
\lambda(a b) & =\sum a_{0} b_{0} \otimes a_{1} b_{1} \text { for } a, b \in R ; \\
\lambda(1) & =1 \otimes 1 .
\end{aligned}
$$

See [Mo], Section 4.1 for more details. In the particular case of the group algebra, $R$ is a $k G$-comodule algebra if and only if it is $G$-graded as an algebra.

If $\epsilon$ is the augmentation for $H$ then those functionals which are $\epsilon$-derivations,

$$
\operatorname{Der}_{k}^{\epsilon}(H, k)=\left\{f \in H^{*} \mid f(a b)=\epsilon(a) f(b)+f(a) \epsilon(b)\right\},
$$


comprise a Lie algebra under the commutator $[f, g]=f * g-g * f$. (Here $*$ is convolution on $H^{*}$.) When $H=k G$ then

$$
\operatorname{Der}_{k}^{\epsilon}(k G, k)=\operatorname{Hom}\left(G, k^{+}\right) .
$$

Back to the general set-up, for each $f \in \operatorname{Der}_{k}^{\epsilon}(H, k)$ define $D_{f} \in \operatorname{Hom}_{k}(R, R)$ by

$$
D_{f}(a)=\sum f\left(a_{0}\right) a_{1}
$$

for all $a \in R$. We leave it as an exercise that $D_{f} \in \operatorname{Der}(R)$ and the map $\operatorname{Der}_{k}^{\epsilon}(H, k) \rightarrow \operatorname{Der}(R)$ sending $f$ to $D_{f}$ is a Lie algebra homomorphism. This construction subsumes our earlier $D_{\chi}$.

The subspace of derivations spanned by all $D_{f}$, as one runs over all comodule algebra actions of one or more Hopf algebras $H$, deserves future scrutiny.

\section{References}

[AP] I. Assem and J.A. de la Peña, The fundamental groups of a triangular algebra, Comm. in Algebra, 24(1) (1996), 187-208.

[BM] M.J. Bardzell and E.N. Marcos, Induced boundary maps for the cohomology of monomial and Auslander algebras, Algebras and Modules II, VIII ICRA, CMS Conf. Proc., 24 (1998).

[FGGM] D.R. Farkas, Ch. Geiss, E.L. Green and E.N. Marcos, Diagonalizable derivations of finite-dimensional algebras I, Israel J. of Math., to appear.

[G] E.L. Green, Graphs with relations, coverings, and group-graded algebras, Trans. AMS, 279(1) (1983), 297-310.

[Mo] Susan Montgomery, Hopf Algebras and Their Actions on Rings, CBMS, 82, Amer. Math. Soc., Providence, 1993.

[S] A. Skowroński, Simply connected algebras and Hochschild cohomologies, Representations of Algebras, Ottawa ‘92, CMS Conf. Proc., 14 (1993).

Received January 5, 1999. The research of Green was partially supported by a grant from NSA. Research of Marcos was partially suported by Fapesp Post-Doctoral Scholarship, (processo 1997/3313-5), and CNPq Research Scholarship, (process 30656/88-0).

\section{Department of Mathematics}

Virginia Polytechnic Institute and State University

BLACKSBURG, VA 24061

E-mail address: farkas@math.vt.edu 
E-mail address: green@math.vt.edu

Instituto de Matemática e Estatística

Universidade de SÃo Paulo, CP 66281

05389-970 SÃo PAUlO SP

BRASIL

E-mail address: enmarcos@ime.usp.br 